\title{
Status of ovarian tissue cryopreservation for adolescent and young adult patients with cancer in Hyogo College of Medicine College Hospital
}

\author{
Wakimoto Y*, Ukita Y, Fuji Y, Wakimoto G, Fukui A and Shibahara $H$ \\ Department of Obstetrics and Gynecology, Hyogo College of Medicine, Nishinomiya, Hyogo, Japan
}

\begin{abstract}
Objective: Cancer treatment for adolescent and young adult (AYA) patients with cancer can have lasting effects on their reproductive health by causing premature menopause. Fertility can be preserved by cryopreservation of oocytes, embryos, or ovarian tissue. Recently, many cases of pregnancy and delivery after ovarian tissue warming and transplantation have been reported. The number of ovarian tissue cryopreservation cases has been increasing in our hospital. Herein, we evaluated the current status of ovarian tissue cryopreservation among AYA patients.
\end{abstract}

Method: Ovarian tissue cryopreservation was performed in nine cases at our hospital between February 2017 and March 2018. In 6 of the 9 patients, oocyte pick-up without controlled ovarian stimulation was performed for both ovaries during the removal of one ovary by laparoscopic surgery. We retrospectively collected data from the patients' medical records.

Result: The patients ranged in age from 11 to 43 years, and all the patients were nulligravida. Among these patients, 3 had breast cancer; 2, leukemia; 1, Ewing sarcoma; 1, vulvar sarcoma; 1, mediastinal tumour; and 1, suprasellar germ cell tumour. Five of the 9 patients underwent ovarian tissue cryopreservation after chemotherapy. The mean anti-Müllerian hormone (AMH) level was $1.54 \mathrm{ng} / \mathrm{ml}$. Twenty-six oocytes were collected from 6 patients. Ten oocytes matured in vitro could be cryopreserved.

Conclusion: So far, there have been no cases of pregnancy by ovarian tissue warming and transplantation. Further accumulation of cases and consideration is needed to yield any findings on the usefulness of this technique.

\section{Introduction}

Cancer treatment in adolescent and young adult (AYA) patients with cancer can have lasting effects on their reproductive health by causing premature menopause $[1,2]$. Fertility can be preserved by cryopreservation of oocytes, embryos, or ovarian tissue $[1,3]$.

At our institution, we have been performing fertility preservation by cryopreservation for AYA patients with cancer since 2013 [3]. Recently, many cases of pregnancy and delivery after ovarian tissue warming and transplantation have been reported [1,4]. The number of ovarian tissue cryopreservation cases in our hospital has been increasing.

Here, we evaluated the status of ovarian tissue cryopreservation in AYA patients with cancer in Hyogo College of Medicine College Hospital.

\section{Materials and methods}

Ovarian tissue cryopreservation was performed in 9 cases at our hospital between February 2017 and August 2017. Vitrification of the ovarian tissue was performed using the cryotissue method (Cryotissue Kit; Kitazato BioPharma Co., Ltd., Shizuoka, Japan). In 6 of 9 patients, oocyte pick-up (OPU) without controlled ovarian stimulation was performed during removal of one ovary under laparoscopic surgery. The contents of small follicles on the ovarian surface are aspirated under laparoscopic surgery by using a suction needle (Hakko Medical Co. Ltd., Tokyo, Japan) equipped with a syringe to retrieve oocytes from one ovary. The contents of the small follicle on the ovarian surface are manually aspirated from another removed ovary by using a $23-\mathrm{G}$ needle equipped with a syringe. Immature oocytes were then incubated in Sage IVM medium (Cooper Surgical, Trumbull, CT, USA) and matured in vitro to the MII stage. Mature oocytes obtained from IVM were cryopreserved using the Cryotop method (Kitazato BioPharma Co., Ltd., Fuji, Shizuoka, Japan).

We retrospectively collected data from medical records and analysed age, gravidity or parity, marriage, primary disease, previous treatment, anti-Müllerian hormone (AMH) levels, OPU, the number of oocytes collected, the number of cryopreserved oocytes, hospitalization period, the number of days from first visit to operation, and the number of fertility preservation cases by cryopreservation of oocytes, embryos, or ovarian tissue.

\section{Result}

Figure 1 shows the comparison between the number of cycles and fertility preservation techniques by cryopreservation of oocytes, embryos, or ovarian tissue. Fertility preservation was performed in

*Correspondence to: Wakimoto Y, Department of Obstetrics and Gynecology, Hyogo College of Medicine, Mukogawa-cho, Nishinomiya, Hyogo, Japan, Tel: +81798456481; Fax: +81798464163; E-mail: yuuwakimoto@gmail.com

Key words: fertility preservation, ovarian tissue cryopreservation, IVM, AYA

Received: April 04, 2018; Accepted: April 22, 2018; Published: April 25, 2018 
7 cycles in 2018 (January to March), 20 cycles in 2017, 18 cycles in 2016,15 cycles in 2015, three cases in 2014, and one case in 2013. The first ovarian tissue cryopreservation under medical indication for an unmarried woman with breast cancer was performed at our centre in February 2017. The distributions of fertility preservation cases with oocytes, embryos, and ovarian tissue cryopreservation were as follows respectively: 1,0 , and 0 in 2013; 3, 0 , and 0 in 2014; 13,2, and 0 in 2015; 9, 8, and 0 in 2016; 6, 6, and 7 in 2017; and 3, 2, and 2 in 2018 (January to March). In 2016, split cryopreservation, which includes both embryo and oocyte freezing in an OPU cycle, was performed in 1 case.

Table 1 shows 9 cases of ovarian tissue cryopreservation. The patients' ages ranged from 11 to 43 years, and all patients were nulligravida. Among these patients, 3 had breast cancer; 2 had leukemia; 1 had Ewing sarcoma; 1 had vulvar sarcoma; 1 had mediastinal tumour; and 1 had suprasellar germ cell tumour. Five of the 9 patients had their ovarian tissue cryopreserved after chemotherapy. The mean AMH level was $1.54 \mathrm{ng} / \mathrm{ml}$. Twenty-six oocytes were collected from 6 patients. Ten oocytes matured in vitro could be cryopreserved. The mean length of hospitalization was 5.11 days. The mean interval from first visit to operation was 16.3 days.

\section{Discussion}

The world's first live birth after cryopreserved ovarian tissue transplantation was reported in a woman with stage IV Hodgkin lymphoma in Belgium in 2004 [5] . According to this report, in 1997, ovarian tissue was taken and cryopreserved before cancer treatment.
Following this, the patient had premature ovarian insufficiency. In 2003, transplantation of thawed ovarian tissue was performed using laparoscopy. The first pregnancy after this procedure was reported in 2004; since then, the pregnancy and live-birth rates have continued to increase steadily and have been exponentially increasing in the past 2 years [1]. Although the number of transplantations performed worldwide is unknown, the reported number of live births by ovarian tissue cryopreservation and transplantation is $>130$ cases as of June 2017 , with pregnancy and live-birth rates being approximately $30 \%$ and $25 \%$, respectively [1].

At our hospital, ovarian tissue cryopreservation was performed in 9 cases. A center for reproductive medicine was established in 2013 at our hospital. A regional oncofertility network of patients, oncologists, and fertility specialists in Gifu prefecture (GPOFS) was established for the first time in Japan in February 2013 [6]. This oncofertility network is a medical institution cooperation system to provide information related to oncofertility counselling for AYA patients with cancer [7]. In Hyogo prefecture, the Hyogo oncofertility network (Hyogo-OFN) was established in collaboration with the Hyogo Society of Obstetrics and Gynaecology and Hyogo Prefecture Regional Cooperation Conference of Cancer Management in January 2016 [3]. Therefore, the number of young women with cancer who received fertility preservation increases every year in our hospital. The number of fertility preservation cases by means of ovarian tissue cryopreservation has been increasing since 2017. So far, at our hospital, none of the cases has requested the use of ovarian tissue warming and transplantation.
(No. of cycles)

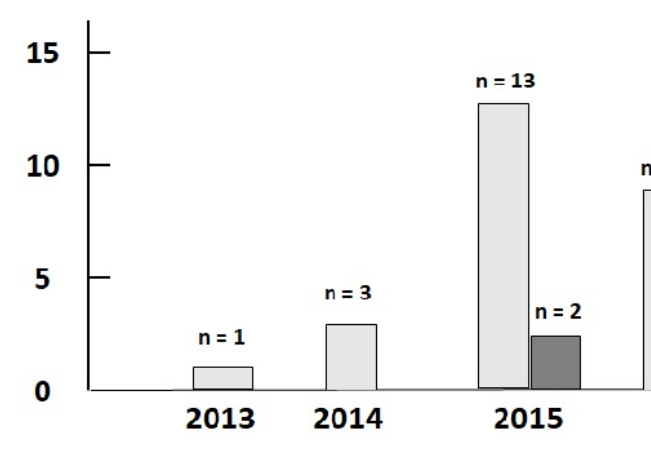

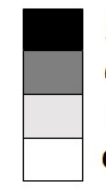

Split cryopreservation Oocyte cryopreservation Embryo cryopreservation Ovarian tissue cryopreservation

Figure 1. Comparison of the number of cycles for fertility preservation between the 4 groups from 2013 to 2018 (January to March).

Table 1.9 cases of ovarian tissue cryopreservation

\begin{tabular}{|c|c|c|c|c|c|c|c|c|c|c|c|}
\hline CASE & $\underset{\mathbf{y r}}{\mathrm{AGE}}$ & $\mathbf{G} / \mathbf{P}$ & Marital status & $\begin{array}{l}\text { AMH } \\
\text { ng/ml }\end{array}$ & $\begin{array}{l}\text { Primary } \\
\text { disease }\end{array}$ & $\begin{array}{l}\text { Previous } \\
\text { treatment }\end{array}$ & $\begin{array}{l}\text { Treatment } \\
\text { plan }\end{array}$ & OPU & Freeze & $\begin{array}{c}\text { Hospitalization period } \\
\text { (days) }\end{array}$ & $\begin{array}{l}\text { From first visit } \\
\text { to operation } \\
\text { (days) }\end{array}$ \\
\hline 1 & 11 & G0 & unmarried & 2.0 & Mediastinal tumor & Chemo & Chemo+HSCT & 5 & MII 1 & 5 & 4 \\
\hline 2 & 15 & G0 & unmarried & 1.12 & Vulvar cancer & Chemo+ope & Chemo & 3 & 0 & 5 & 11 \\
\hline 3 & 14 & G0 & unmarried & 0.23 & AML recurrence & Chemo & Chemo+HSCT & 1 & 0 & 5 & 4 \\
\hline 4 & 15 & G0 & unmarried & 0.13 & ALL recurrence & Chemo & Chemo+HSCT & 1 & 0 & 5 & 8 \\
\hline 5 & 16 & G0 & unmarried & 0.03 & Ewing sarcoma & Chemo & Ope+chemo & Not done & & 5 & 22 \\
\hline 6 & 42 & G0 & unmarried & 0.44 & Breast cancer & No & Ope+chemo & Not done & & 6 & 27 \\
\hline 7 & 33 & G0 & unmarried & 2.16 & $\begin{array}{l}\text { Breast cancer } \\
\text { (Lynch syn.) }\end{array}$ & No & Ope+chemo & Not done & & 5 & 48 \\
\hline 8 & 43 & G0 & unmarried & 1.63 & Breast cancer & No & Ope+chemo & 9 & MII 5 & 5 & 20 \\
\hline 9 & 14 & G0 & unmarried & 6.11 & $\begin{array}{l}\text { Suprasellar germ cell } \\
\text { tumor }\end{array}$ & No & chemo & 7 & MII 4 & 5 & 3 \\
\hline
\end{tabular}


Currently, the fertility of AYA women with cancer can be preserved through oocyte, embryo, or ovarian tissue cryopreservation [1]. Embryo cryopreservation requires sperm from the husband or partner. In the case of mature oocyte and ovarian cryopreservation, sperm is not needed. However, both embryo cryopreservation and mature oocyte cryopreservation might delay cancer treatment because of controlled ovarian stimulation (COS). Conversely, ovarian cryopreservation avoids COS, which is needed in embryo or oocyte cryopreservation. As a result, cancer treatment can be initiated immediately and can be indicated for pubertal girls before starting their menstrual period. Therefore, ovarian cryopreservation could be the only option for fertility preservation in prepubertal patients [1]. In addition, this method allows the storage of a large number of follicles. However, when metastasis of the tumour cells in the ovary is involved, that is, minimal residual disease (MRD) [8], transplantation of thawed ovarian tissue is associated with the risk of recurrence. Especially patients with leukemia are at high risk for transfer of malignant cells with the transplanted tissue [9]. In our hospital, we have performed fertility preservation through ovarian tissue cryopreservation for 2 AYA women with leukemia. After the transplantation of cryopreserved ovarian tissue, these AYA women may be at increased risk of cancer relapse because of ovarian tissue contamination by leukemic cells. As alternative approaches to auto-transplantation, in vitro maturation of primordial follicles or their transplantation in an artificial ovary is assumed to be necessary [10-13]. However, they are yet to be applicable in humans and cannot be offered to patients [9].

In November 2017, the first delivery in a leukemia survivor after transplantation of cryopreserved ovarian tissue was reported [9]. This case report suggests that transplantation safety should be increased through comprehensive tissue evaluation for malignant contamination when transplantation is considered in leukemia survivors. Moreover, tissue collection is recommended during the safest time possible, preferably during complete remission [9]. In our hospital, ovarian tissue samples were obtained from 2 AYA women with leukemia during complete remission before hematopoietic stem cell transplantation (HSCT), and cryopreservation of the ovary was performed. In addition, as the use of frozen oocytes matured in vitro should avoid this risk, we performed cryopreservation of in vitro matured oocytes in addition to ovarian tissue cryopreservation [14]. Six of the 9 patients were treated with OPU during ovarian tissue cryopreservation. Twenty-six oocytes were collected, and $10 \mathrm{MII}$ oocytes were frozen.

In cases 1-5, the AMH level was low despite that the patients were in their teens. However, the AMH level immediately after the end of cancer therapy was reported to be inappropriate for the evaluation of the ovarian reserve [15]. At 11 years after a successful allogeneic bone marrow transplantation (BMT), a patient became pregnant and delivered a healthy infant in full term [16].

In conclusion, so far, at our hospital, there have been no cases of pregnancy by ovarian tissue warming and transplantation. As the success rate of births in women transplanted with frozen-thawed ovarian tissue is low worldwide [1], further accumulation of cases and consideration is needed to yield findings on the usefulness of this technique. However, we believe that fertility preservation of ovarian tissue through cryopreservation will help AYA patients fight cancer by providing hope that they can conceive in the future.

\section{References}

1. Donnez J, Dolmans MM (2018) Fertility Preservation in Women. N Engl J Med 378: 400-401. [Crossref]

2. Suzuki N (2018) Clinical Practice Guidelines for Fertility Preservation in Pediatric, Adolescent, and Young Adults with Cancer. Int J Clin Oncol 1269-1274 [Crossref]

3. Wakimoto Yu, Akatani-Hasegawa Akiko, Wakimoto Goh, Ukita Misato, Sugiyama Yukiko, et al. (2017) Fertility Preservation for Adolescent and Young Adult Women in Hyogo College of Medicine College Hospital. ACTA MEDICA HYOGOENSIA 42 101-107.

4. Gellert SE, Pors SE, Kristensen SG, Bay-Bjørn AM, Ernst E, et al. (2018) Transplantation of frozen-thawed ovarian tissue: an update on worldwide activity published in peer-reviewed papers and on the Danish cohort. $J$ Assist Reprod Genet. [Crossref]

5. Donnez J, Dolmans MM, Demylle D, Jadoul P, Pirard C, et al. (2004) Livebirth after orthotopic transplantation of cryopreserved ovarian tissue. Lancet 364: 1405-1410. [Crossref]

6. Furui T, Takenaka M, Makino H, Terazawa K, Yamamoto A, et al. (2016) An evaluation of the Gifu Model in a trial for a new regional oncofertility network in Japan, focusing on its necessity and effects. Reprod Med Biol 15: 107-113. [Crossref]

7. Suzuki N (2016) Oncofertility in Japan: advances in research and the roles of oncofertility consortia. Future Oncol 12: 2307-2311. [Crossref]

8. Practice Committee of American Society for Reproductive Medicine (2014) Ovarian tissue cryopreservation: a committee opinion. Fertil Steril 101: 1237-1243. [Crossref]

9. Shapira M, Raanani H, Barshack I, Amariglio N, Derech-Haim S, et al. (2018) First delivery in a leukemia survivor after transplantation of cryopreserved ovarian tissue, evaluated for leukemia cells contamination. Fertil Steril 109: 48-53. [Crossref]

10. Ikeda Y, Hasegawa A, Tsubamoto H, Wakimoto Y, Kumamoto K, et al. (2016) Effects of gremlin-2 on the transition of primordial follicles during early folliculogenesis in the human ovary. Eur J Obstet Gynecol Reprod Biol 203: 72-77. [Crossref]

11. Mahmoud Salama, Teresa K Woodruff (2015) New advances in ovarian autotransplantation to restore fertility in cancer patients. Cancer Metastasis Rev 34: 807-822. [Crossref]

12. Chiti MC, Dolmans MM, Lucci CM, Paulini F, Donnez J, et al. (2017) Further insights into the impact of mouse follicle stage on graft outcome in an artificial ovary environment. Mol Hum Reprod 23: 381-392. [Crossref]

13. Hasegawa A, Mochida N, Ogasawara T, Koyama K (2006) Pup birth from mouse oocytes in preantral follicles derived from vitrified and warmed ovaries followed by in vitro growth, in vitro maturation, and in vitro fertilization. Fertil Steril 86: 1182-1192. [Crossref]

14. Yin H, Jiang H, Kristensen SG, Andersen CY (2016) Vitrification of in vitro matured oocytes collected from surplus ovarian medulla tissue resulting from fertility preservation of ovarian cortex tissue. J Assist Reprod Genet 33: 741-746. [Crossref]

15. Miyoshi Y, Yasuda K, Tachibana M, Yoshida H, Miyashita E, et al. (2016) Longitudinal observation of serum anti-Müllerian hormone in three girls after cancer treatment. Clin Pediatr Endocrinol 25: 119-126. [Crossref]

16. Toshiki Kawahara (2001) Successful Pregnancy after Allogeneic Bone Marrow Transplantation Acute Promyelocytic Leukemia. ACTA OBEST GYNAEC JPN.

Copyright: (C2018 Wakimoto Y. This is an open-access article distributed under the terms of the Creative Commons Attribution License, which permits unrestricted use, distribution, and reproduction in any medium, provided the original author and source are credited. 